\title{
An examination of food security and fruit and vegetable consumption using the Food and You Survey
}

\author{
O. Turnbull and H. Ensaff \\ School of Food Science and Nutrition, University of Leeds, LS2 9JT.
}

Food security is dependent on the accessibility, affordability and utilization of food ${ }^{(1)}$ and can be influenced by socioeconomic factors such as education, working status, age, gender and household income ${ }^{(2)}$. In the UK, 4.7 million people are unable to eat food of an adequate quantity or of sufficient nutritious quality, partly due to food insecurity ${ }^{(3)}$. This study aimed to explore the relationship between food security and fruit and vegetable consumption.

Data for a representative sample $(n=3118)$ aged $\geqslant 16$ years living in England, Wales and Northern Ireland were obtained from the Food and You Survey ${ }^{(4)}$. Analysis focussed on items related to food security and fruit and vegetable consumption. This included items from a validated measure of food security ${ }^{(5)}$ used by the U.S. Department of Agriculture (USDA), e.g. 'In the last 12 months, did you lose weight because there wasn't enough money for food?' Other items related to specific changes made to food habits due to financial reasons, e.g. 'Bought items that were on special offer more?' Fruit and vegetable consumption was established by 3 items in the survey, e.g. 'How often do you eat cooked vegetables?'

A 'food security score' was created from the 10 relevant items, with a lower score corresponding to a higher level of food security. A 'food changes score' was created from items relating to financially-driven food changes, with a lower score reflecting fewer changes. In addition, a 'fruit and vegetable score' was generated from the items related to fruit and vegetable consumption, with a lower score corresponding to lower consumption.

Kendall's tau tests were performed and a statistically significant negative correlation between food security and fruit and vegetable consumption $(\tau=-0 \cdot 116, p<0 \cdot 001)$ and between food changes and fruit and vegetable consumption $(\tau=-0 \cdot 043, p=0 \cdot 002)$ was found. Logistic regression analysis was carried out to assess the relationship between food security and food changes and fruit and vegetable consumption (dichotomised to low and high). The logistic model for fruit and vegetable consumption was adjusted for gender, age, household income, education, working status and country. The full model was statistically significant $(p<0 \cdot 0005)$ and correctly classified $65.2 \%$ of cases and explained $18.5 \%$ (Nagelkerke $\mathrm{R}^{2}$ ) of the variance in fruit and vegetable consumption. Food security and food changes, independently, were significant predictors $(p=0.001$ and $p=0 \cdot 013$, respectively) for fruit and vegetable consumption. With every unit increment in the food security score, there is an $11 \%$ decrease in the odds of being a high fruit and vegetable consumer. Likewise, the odds of being a high fruit and vegetable consumer decreases by $5 \%$ with every increment in the food changes score.

In conclusion this study showed that food security, including changes to food habits, were significantly associated with fruit and vegetable consumption. Research is needed to examine further the relationship between food security and fruit and vegetable consumption. In addition, further work should be carried out into food security and potential associations with other elements of the diet.

1. Bach C \& Aborisade B (2014) Assessing the Pillars of Sustainable Food Security. European International Journal of Science and Technology 3, 117-125.

2. Johnston JL, Fanzo JC \& Cogill B (2014) Understanding Sustainable Diets: A Descriptive Analysis of the Determinants and Processes That Influence Diets and Their Impact on Health, Food Security, and Environmental Sustainability. Adv Nutr 5, 418-429.

3. Garthwaite KA, Collins PJ \& Bambra C (2015) Food for thought: An ethnographic study of negotiating ill health and food insecurity in a UK foodbank. Soc Sci \& Med 132, 38-44.

4. Bates B, Hovard P, Lubian K et al. (2017) Food and You Survey Wave 4 Technical Report.

5. Bickel G, Nord M, Price C et al. (2000) Guide to Measuring Household Food Security Revised. Measuring Food Security in the United States: Reports of the Federal Interagency Food Security Measurement Project no. 6. 